九州大学学術情報リポジトリ

Kyushu University Institutional Repository

\title{
AN EXPLICIT FORMULA OF THE SHAPLEY VALUE FOR THE CONJUGATE-POINT GAME
}

\section{Fuchikami, Takeaki}

Nissay Information Technology Co. Ltd

Kawasaki, Hidefumi

Faculty of Mathematics, Kyushu University

https://doi.org/10.5109/1906487

出版情報: Bulletin of informatics and cybernetics. 47, pp.11-24, 2015-12. Research Association of Statistical Sciences

バージョン:

権利関係 : 


\title{
AN EXPLICIT FORMULA OF THE SHAPLEY VALUE FOR THE CONJUGATE-POINT GAME
}

\author{
By \\ Takeaki FUCHIKAMI* and Hidefumi KAWASAKI ${ }^{\dagger}$
}

\begin{abstract}
The conjugate point was introduced by Jacobi to derive a sufficient optimality condition for a variational problem. One of the authors defined the conjugate point for an extremal problem in $\mathbb{R}^{n}$. The key of the conjugate point is a coalition of variables. Namely, when there exists a conjugate point for a stationary solution $x \in \mathbb{R}^{n}$, the solution is improved by suitably changing some of the variables. This fact leads us to a cooperative game. One of the solution concepts for cooperative games is the Shapley value. It evaluates player's contribution in the cooperative game. However, its calculation is usually very hard. The purpose of this paper is to provide a cooperative game, which we call the conjugate-point game, whose Shapley value can be explicitly computed.
\end{abstract}

Key Words and Phrases: Shapley value, cooperative game, conjugate-point game, conjugate point.

\section{Introduction}

The conjugate point was originally introduced to guarantee local optimality of a stationary solution $x(t)$ for the simplest problem in the calculus of variations

$$
\begin{array}{ll}
\text { Minimize } & \int_{0}^{T} f(t, x(t), \dot{x}(t)) d t \\
\text { subject to } & x(0)=A, x(T)=B
\end{array}
$$

where $A$ and $B$ are given points, see e.g. Gelfand and Fomin (1963). Kawasaki (2000, 2001) defined the conjugate point for an extremal problem with $n$ variables

$$
\left(P_{0}\right) \quad \text { Minimize } f(x), \quad x \in \mathbb{R}^{n} .
$$

One can find a typical example of the conjugate point for $\left(P_{0}\right)$ in the shortest polygonal path problem on an ellipsoid

$$
\frac{x^{2}}{a^{2}}+\frac{y^{2}}{a^{2}}+\frac{z^{2}}{c^{2}}=1,
$$

where $a>c$. The extremal problem is to find the shortest polygonal path

$$
A=X_{0}, X_{1}, \ldots, X_{n}, X_{n+1}=B
$$

* Nissay Information Technology Co.Ltd, fuchikami1027@gmail.com

$\dagger$ Faculty of Mathematics, Kyushu University, kawasaki@math.kyushu-u.ac.jp 


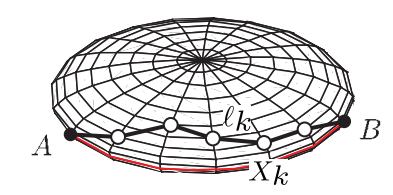

Figure 1: The shortest polygonal path problem

joining two given points $A:=(a, 0,0)$ and $B:=(a \cos T, a \sin T, 0)$, where each $X_{k}$ moves on a longitude $\ell_{i}$ equally located between $A$ and $B$. Since each $X_{k}$ has one degree of freedom, this problem is formulated as $\left(P_{0}\right)$, and the equatorial polygonal path is a stationary solution for $\left(P_{0}\right)$. Further, whether the stationary solution is minimal or not depends on $T$. According to Kawasaki (2001), it is minimal when $T<a \pi / c$, and not minimal when $T>a \pi / c$ and $n$ is sufficiently large. In the latter case, we call the first number $k$ satisfying $(k+1) T /(n+1)>a \pi / c$ a strict conjugate point, which matches the classical conjugate point. When there exists a strict conjugate point $k \leq n$, the equatorial polygonal path is not a local minimum.

In general, let $x=\left(x_{1}, \ldots, x_{n}\right)$ be a stationary solution for $\left(P_{0}\right)$, that is, the gradient vector $f^{\prime}(x)$ vanishes. Let $\left(a_{i j}\right):=f^{\prime \prime}(x)$ the Hessian matrix of $f$ at $x$. According to Sylvester's criterion, if $f^{\prime \prime}(x)$ has a negative leading principal minor $\operatorname{det}\left(a_{i j}\right)_{1 \leq i, j \leq k}$, then $x$ is not a minimal solution. So it can be improved by suitably changing some variables as

$$
f\left(x_{1}, \ldots, x_{k}, x_{k+1}, \ldots, x_{n}\right)>f\left(x_{1}+y_{1}, \ldots, x_{k}+y_{k}, x_{k+1}, \ldots, x_{n}\right)
$$

for some small variation $\left(y_{1}, \ldots, y_{k}\right)$. Here we emphasize that a single variation $y_{i}$ is not enough to improve $x$, we need a coalition of variables. This fact leads us to a cooperative game. One of the solution concepts for cooperative games is the Shapley value defined by Shapley (1953). It evaluates player's contribution in the cooperative game. However, its calculation is usually very hard.

The aims of this paper are to define a cooperative game based on coalition of variables, and to present an explicit formula of the Shapley value for this game.

\section{Definitions and Notations}

In this section, we first define the conjugate-point game, which is induced from the shortest path problem on the ellipsoid (1). Next, we define two sets $I(i ; S)$ and $\operatorname{Ker}(i ; S)$ to compute the Shapley value.

Let $N=\{1, \ldots, n\}$ be the set of players and $k \in N$. In the following $k$ means the least number of required sequential players to improve $x$. We call

$$
[j: j+k-1]:=\{j, j+1, \ldots, j+k-1\} \subset N
$$

an interval of length $k$. For any subset $S$ of $N$, we define a characteristic function $v(S)$ as the maximum number of disjoint intervals of length $k$ contained in $S$. We call this cooperative game the conjugate-point game and denote it by $G(n, k)$. we put

$$
n=p k+r(0 \leq r<k),
$$




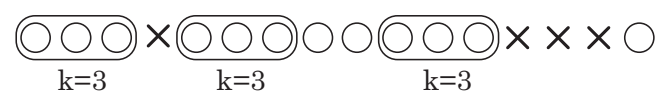

Figure 2: When $S$ consists of the circles, it holds that $v(S)=3$.

and denote by $\phi_{i}(n, k)$ the Shapley value of $G(n, k)$, that is,

$$
\phi_{i}(n, k)=\sum_{i \in S \subset N} \frac{(s-1) !(n-s) !}{n !}\{v(S)-v(S-\{i\})\},
$$

where $s:=\# S$. The following expression is well-known, see e.g. Aumann et al (1992).

$$
\phi_{i}(n, k)=\sum_{\pi \in \Pi} \frac{1}{n !}\left\{v\left(S_{\pi, i}\right)-v\left(S_{\pi, i}-\{i\}\right)\right\},
$$

where $\Pi$ denotes the set of all permutation on $N$ and $S_{\pi, i}$ denotes the set of player $i$ and players preceding $i$ with respect to $\pi$, that is,

$$
S_{\pi, i}=\{j \mid \pi(j) \leq \pi(i)\} .
$$

It is evident from symmetry of $v(S)$ that $\phi_{i}=\phi_{n-i+1}$ for any $i \in N$. We call any element of the following set a pivot of $S$. Pivots are regarded as key players in $S$.

$$
W_{S}:=\{i \mid v(S)-v(S-\{i\})=1\} .
$$

Then the Shapley value (5) is simply written as

$$
\phi_{i}=\frac{1}{n !} \#\left\{\pi \mid i \in W_{S_{\pi, i}}\right\} .
$$

So it suffices to test whether $i \in S$ is a pivot of $S$ or not in order to compute $\phi_{i}$.

Definition 2.1. For any $i \in N$ and $S \subset N$ including $i$, we denote by $I(i ; S) \subset S$ the maximum interval including $i$. We denote by $\operatorname{Ker}(i ; S)$ the remainder of $I(i ; S)$ after removing intervals of length $k$ from both sides of $i$ as much as possible with keeping $i$.

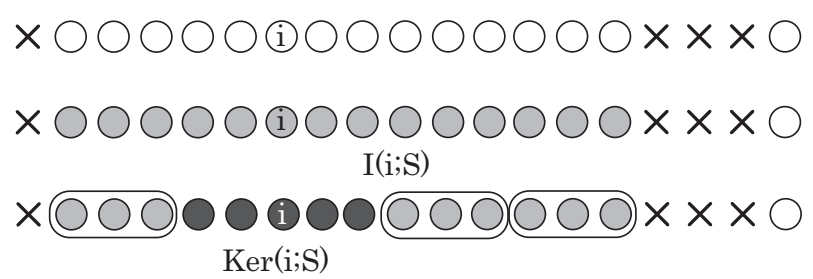

Figure 3: When $k=3$ and $S$ consists of white circles, $I(i ; S)$ consists of gray circles and $\operatorname{Ker}(i ; S)$ consists of black cirlces. 
Lemma 2.2. For any $i \in S$, it holds that \#Ker $(i ; S) \leq 2 k-1$. Furthermore the following conditions are equivalent to each others. (i) $i \in W_{S}$, (ii) $i \in W_{I(i ; S)}$, (iii) $i \in W_{\operatorname{Ker}(i ; S)}$, (iv) $\# \operatorname{Ker}(i ; S) \geq k$.

Proof. The first claim and (i) $\Leftrightarrow$ (ii) are evident from Figure 3. (ii) $\Leftrightarrow$ (iii): It is enough to consider the case that $\left[j_{1}: j_{2}\right]:=I(i ; S) \neq \operatorname{Ker}(i ; S)$. By virtue of symmetry, we may assume that $j_{1}+k \leq i$. Then, since $v\left(\left[j_{1}: j_{2}\right]\right)=v\left(\left[j_{1}+k: j_{2}\right]\right)+1$ and $v\left(\left[j_{1}: j_{2}\right]-\{i\}\right)=v\left(\left[j_{1}+k: j_{2}\right]-\{i\}\right)+1$,

$$
\begin{aligned}
i \in W_{I(i ; S)} & \Leftrightarrow v\left(\left[j_{1}: j_{2}\right]\right)-v\left(\left[j_{1}: j_{2}\right]-\{i\}\right)=1 \\
& \Leftrightarrow v\left(\left[j_{1}+k: j_{2}\right]\right)-v\left(\left[j_{1}+k: j_{2}\right]-\{i\}\right)=1 \\
& \Leftrightarrow \quad i \in W_{I(i ; S)-\left[j_{1}: j_{1}+k-1\right]} .
\end{aligned}
$$

Repeating this procedure, we get $\operatorname{Ker}(i ; S)$ as the remainder and see the equivalence of (ii) and (iii). (iii) $\Leftrightarrow$ (iv): Since we cannot remove any interval of length $k$ from $\operatorname{Ker}(i ; S)$ without deleting $i$, this assertion is clear.

\section{The Shapley value of player 1}

In this section, we compute the Shapley value $\phi_{1}$. It is clear from Lemma 2.2 that

$$
1 \in W_{S} \Leftrightarrow \# \operatorname{Ker}(1 ; S) \geq k \Leftrightarrow \operatorname{Ker}(1 ; S)=[1: k]
$$

Since $n=p k+r$ and $I(1 ; S)$ is obtained by adding disjoint intervals of length $k$ to $\operatorname{Ker}(1 ; S)$, we get from $(9)$ that if $i \in W_{S}, I(1 ; S)$ is expressed as

$$
1 \leq \exists m \leq p \text { s.t. } I(1 ; S)=[1: m k]
$$

Lemma 3.1. Let $1 \leq m \leq p$ and $\pi \in \Pi$. Then $I\left(1 ; S_{\pi, 1}\right)=[1: m k]$ if and only if

$$
\pi(j)<\pi(1) \quad 2 \leq \forall j \leq m k
$$

and either $(\mathrm{a}) \pi(m k+1)>\pi(1)$ or $(\mathrm{b}) m k=n$ holds, $($ so that $m=p)$.

Proof. Necessity: Since 1 joins $I\left(1 ; S_{\pi, 1}\right)=[1: m k]$ last, $(11)$ is clear. If $m k<n$ and $m k+1$ joins $S_{\pi, 1}$ before 1 , then the interval $I\left(1 ; S_{\pi, 1}\right)$ contains $[1: m k+1]$. Sufficiency is evident.

THEOREM 3.2. Let $n=p k+r(0 \leq r<k)$, then

$$
\phi_{1}= \begin{cases}\sum_{m=1}^{p-1} \frac{1}{m k(m k+1)}+\frac{1}{p k} & \text { if } r=0, \\ \sum_{m=1}^{p} \frac{1}{m k(m k+1)} & \text { if } r \neq 0 .\end{cases}
$$

Proof. By (8), it suffices to compute $\#\left\{\pi \mid 1 \in W_{S_{\pi, 1}}\right\}$. It follows from (9), (10) and Lemma 3.1 that

$$
\begin{aligned}
& \#\left\{\pi \mid 1 \in W_{S_{\pi, 1}}\right\} \\
= & \sum_{m=1}^{p} \#\left\{\pi \mid I\left(1 ; S_{\pi, 1}\right)=[1: m k]\right\} \\
= & \sum_{m=1}^{p} \#\{\pi \mid \pi \text { satisfies }(11) \text { and }(a)\}+\#\{\pi \mid \pi \text { satisfies }(11) \text { and }(b)\} .
\end{aligned}
$$


For each $m$, the first term of (13) is given by

$$
\left(\begin{array}{c}
n \\
m k+1
\end{array}\right)(n-m k-1) !(m k-1) !=\frac{n !}{m k(m k+1)} .
$$

Indeed, such a permutation $\pi$ satisfies

$$
\pi(j)<\pi(1)<\pi(m k+1) \quad 2 \leq \forall j \leq m k .
$$

There are $\left(\begin{array}{c}n \\ m k+1\end{array}\right)$ ways to choose $P:=\pi([1: m k+1]) \subset N$. Since $\pi([2: m k])$ can freely share the first $m k-1$ places of $P$, and since the complement of $P$ can be freely shared by other $n-m k-1$ numbers, we get (14). On the other hand, since case (b) occurs only when $\pi(1)=n$ and $m k=p k=n$, (so that $r=0$ ), we similarly see that the second term of (13) is equal to $(n-1)$ !. Hence we get the first result in (12). When $r \neq 0$, since case (b) does not occur, we get the second result in (12).

\section{A recurrence relation of $\left\{\phi_{i}\right\}$ : Case 1}

Starting with $\phi_{1}$, we compute $\phi_{2}, \phi_{3}$, and so on. For this aim, we compute $\phi_{i+1}-\phi_{i}$. Because of symmetry of the game, it suffices to consider the case of $1 \leq i \leq\left[\frac{n-1}{2}\right]$, where [.] denotes Gauss's symbol. Further we divide it into three cases.

Case 1: $n-(k-1) \leq i \leq k-1$, (this is the case that $k \geq \frac{n}{2}+1$ ),

Case 2: $1 \leq i \leq \min \{n-k, k-1\}$,

Case 3: $k \leq i \leq\left[\frac{n-1}{2}\right]$.

Before dealing with Case 1, we present a lemma that is applicable to any case.

LEMma 4.1. $\phi_{i+1}=\phi_{i}+\delta_{i}^{+}-\delta_{i}^{-}$, where

$$
\begin{aligned}
\delta_{i}^{+} & :=\#\left\{\pi \mid i \notin W_{S_{\pi, i+1}}, i+1 \in W_{S_{\pi, i+1}}\right\} / n !, \\
\delta_{i}^{-} & :=\#\left\{\pi \mid i \in W_{S_{\pi, i}}, i+1 \notin W_{S_{\pi, i}}\right\} / n ! .
\end{aligned}
$$

Proof. Since

$$
\begin{aligned}
& n !\left(\phi_{i+1}-\phi_{i}\right) \\
= & \#\left\{\pi \mid i+1 \in W_{S_{\pi, i+1}}\right\}-\#\left\{\pi \mid i \in W_{S_{\pi, i}}\right\} \\
= & \#\left\{\pi \mid i, i+1 \in W_{S_{\pi, i+1}}\right\}+\#\left\{\pi \mid i \notin W_{S_{\pi, i+1}}, i+1 \in W_{S_{\pi, i+1}}\right\} \\
& -\#\left\{\pi \mid i, i+1 \in W_{S_{\pi, i}}\right\}-\#\left\{\pi \mid i \in W_{S_{\pi, i}}, i+1 \notin W_{S_{\pi, i}}\right\},
\end{aligned}
$$

it suffices to prove

$$
\#\left\{\pi \mid i, i+1 \in W_{S_{\pi, i+1}}\right\}=\#\left\{\pi \mid i, i+1 \in W_{S_{\pi, i}}\right\} .
$$

We define a bijection $f: \Pi \rightarrow \Pi$ by $f(\pi):=(i, i+1) \circ \pi$, where $(i, i+1)$ is a transposition. Then, for any $\pi \in \Pi$ such that $i, i+1 \in W_{S_{\pi, i}}$, due to definition of $W_{S}$,

$$
v\left(S_{\pi, i}\right)-v\left(S_{\pi, i}-\{i\}\right)=v\left(S_{\pi, i}\right)-v\left(S_{\pi, i}-\{i+1\}\right)=1 .
$$

Since $S_{\pi, i}=S_{f(\pi), i+1},(17)$ implies that $i, i+1 \in W_{S_{f(\pi), i+1}}$. That is,

$$
f\left(\left\{\pi \mid i, i+1 \in W_{S_{\pi, i}}\right\}\right) \subset\left\{\pi \mid i, i+1 \in W_{S_{\pi, i+1}}\right\} .
$$


Since $f$ is an injection, we have

$$
\#\left\{\pi \mid i, i+1 \in W_{S_{\pi, i}}\right\} \leq \#\left\{\pi \mid i, i+1 \in W_{S_{\pi, i+1}}\right\} .
$$

The converse inequality is similarly obtained.

Let us now consider Case 1.

TheOREM 4.2. For any $i$ such that $n-k+1 \leq i \leq k-1$, it holds that

$$
\delta_{i}^{+}=\delta_{i}^{-}=0 .
$$

Therefore, $\phi_{n-k+1}=\phi_{n-k+2}=\cdots=\phi_{k}$.

Proof. By Lemma 2.2, $i \in W_{S_{\pi, i}}$ if and only if $\# \operatorname{Ker}\left(i ; S_{\pi, i}\right) \geq k$. For such a $\pi$, since $n-k+1 \leq i \leq k-1$ and since we cannot remove any intervals of length $k$ from $\operatorname{Ker}\left(i ; S_{\pi, i}\right)$ without removing $i$, we have

$$
\left\{\operatorname{Ker}\left(i ; S_{\pi, i}\right) \mid i \in W_{S_{\pi, i}}, \pi \in \Pi\right\}=\left\{\begin{array}{llll}
{[1: k],} & {[1: k+1],} & \cdots & {[1: n]} \\
& {[2: k+1],} & \cdots & {[2: n]} \\
& & \ddots & \\
& & & {[n-k+1: n]}
\end{array}\right\} .
$$

We similarly see that $\left\{\operatorname{Ker}\left(i+1 ; S_{\pi, i+1}\right) \mid i+1 \in W_{S_{\pi, i+1}}\right\}$ coincides with set (21). So $\left\{\pi \mid i \in W_{S_{\pi, i}}, i+1 \notin W_{S_{\pi, i}}\right\}$ is empty. (Remark that not $i+1 \notin W_{S_{\pi, i+1}}$ but $i+1 \notin W_{S_{\pi, i}}$. $)$ Indeed, if $\pi$ is an element of this set, then $\operatorname{Ker}\left(i ; S_{\pi, i}\right)$ is one of the intervals in (21) and $i$ is its element. Since $i+1 \leq k, i+1$ belongs to the interval, which implies that $i+1$ is also an element of $S_{\pi, i}$. Since the length of the interval is greater than or equal to $k$, we see from Lemma 2.2 and (21) that $i+1 \in W_{S_{\pi, i}}$. Therefore $\delta_{i}^{-}=0$. Similarly, we have $\delta_{i}^{+}=0$.

\section{A recurrence relation of $\left\{\phi_{i}\right\}$ : Case 2}

In this section, we consider the case that $1 \leq i \leq \min \{n-k, k-1\}$. Since $i \leq k$ and $k+i-1 \leq n$, we get

$$
\left\{\operatorname{Ker}\left(i ; S_{\pi, i}\right) \mid i \in W_{S_{\pi, i}}, \pi \in \Pi\right\}=\left\{\begin{array}{cccc}
{[1: k],} & {[1: k+1],} & \cdots & {[1: k+i-1]} \\
& {[2: k+1],} & \cdots & {[2: k+i-1]} \\
& \ddots & \vdots \\
& & & {[i: k+i-1]}
\end{array}\right\}
$$

as well as (21), where the difference between (21) and (22) is caused from $k+i-1<n$. Since $i+1 \leq k$ and $k+i \leq n$, we similarly see that $\left\{\operatorname{Ker}\left(i+1 ; S_{\pi, i+1}\right) \mid i+1 \in\right.$ $\left.W_{S_{\pi, i+1}}, \pi \in \Pi\right\}$ equals

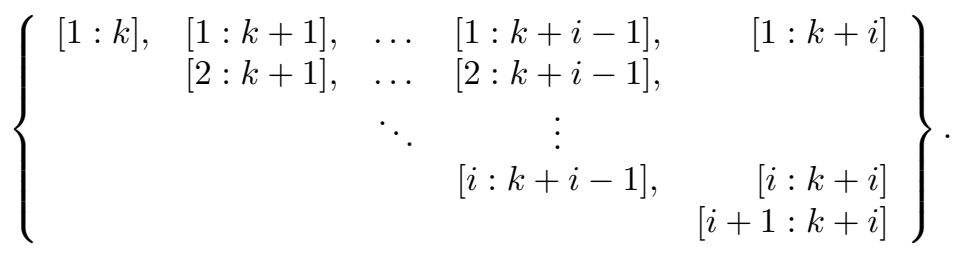


Comparing (22) and (23), we get $\delta_{i}^{-}=0$ as well as Theorem 4.2. On the other hand,

$$
\begin{aligned}
& \left\{\operatorname{Ker}\left(i+1 ; S_{\pi, i+1}\right) \mid i \notin W_{S_{\pi, i+1}}, i+1 \in W_{S_{\pi, i+1}}, \pi \in \Pi\right\} \\
= & \left\{\left[j_{1}: k+i\right] \mid 1 \leq j_{1} \leq i+1\right\} .
\end{aligned}
$$

Indeed, for any interval $\left[j_{1}: k+i\right]\left(1 \leq j_{1} \leq i\right)$ in (23), we can remove the interval $[i+1: k+i]$ with length $k$ from $\left[j_{1}: k+i\right]$ without removing $i$. Then the reminder is $[j: i]$, and its length is less than $k$. Hence it follows from Lemma 2.2 that $i$ is not a pivot of $\left[j_{1}: k+i\right]$. So we get $(24)$.

Since $I\left(i+1 ; S_{\pi, i+1}\right)$ is an interval obtained by adding disjoint intervals of length $k$ to $\operatorname{Ker}\left(i+1 ; S_{\pi, i+1}\right)$, we get from (24) that

$$
\begin{aligned}
& \left\{I\left(i+1 ; S_{\pi, i+1}\right) \mid i \notin W_{S_{\pi, i+1}}, i+1 \in W_{S_{\pi, i+1}}, \pi \in \Pi\right\} \\
= & \left\{\left[j_{1}: m k+i\right] \mid 1 \leq j_{1} \leq i+1, m \geq 1, m k+i \leq n\right\} .
\end{aligned}
$$

Lemma 5.1. Let $m \geq 1$ satisfy $m k+i \leq n$. Then there exists $1 \leq j_{1} \leq i+1$ such that $I\left(i+1 ; S_{\pi, i+1}\right)=\left[j_{1}: m k+i\right]$ if and only if

$$
\pi(j)<\pi(i+1) \quad i+2 \leq \forall j \leq m k+i
$$

and either $(\mathrm{c}) \pi(m k+i+1)>\pi(i+1)$ or $(\mathrm{d}) m k+i=n$ holds.

Proof. Necessity: Since $i+1$ joins $S_{\pi, i+1}$ last, $I\left(i+1 ; S_{\pi, i+1}\right)=\left[j_{1}: m k+i\right]$ implies that (26) and $m k+i+1$ dose not joint $S_{\pi, i+1}$ before $i+1$ if $m k+i<n$. Conversely, it follows from (c) or (d) that any number greater than $m k+i$ does not join $S_{\pi, i+1}$ before $i+1$. Hence $m k+i$ is the maximum number of $I\left(i+1 ; S_{\pi, i+1}\right)$. Since $I\left(i+1 ; S_{\pi, i+1}\right)$ is an interval, it has a form of $\left[j_{1}: m k+i\right]$ for some $1 \leq j_{1} \leq i+1$.

TheOREM 5.2. In the case of $1 \leq i \leq \min \{n-k, k-1\}$, it holds that

$$
\begin{aligned}
\delta_{i}^{+}= \begin{cases}\sum_{\substack{m=1 \\
p-1}}^{p} \frac{1}{m k(m k+1)} & 1 \leq i \leq r-1, \\
\sum_{\substack{m=1 \\
p-1}}^{m k(m k+1)}+\frac{1}{p k} & i=r, \\
\sum_{m=1}^{m k(m k+1)} & r+1 \leq i \leq k-1,\end{cases} \\
\delta_{i}^{-}=0 .
\end{aligned}
$$

Proof. Assume that $\pi$ satisfies that $i \notin W_{S_{\pi, i+1}}$ and $i+1 \in W_{S_{\pi, i+1}}$. Then it is easily seen from (25) and Lemma 5.1 that (26) and either (c) or (d) hold. The number of $\pi$ 's satisfying (26) and (c) is given by

$$
\left(\begin{array}{c}
n \\
m k+1
\end{array}\right)(m k-1) !(n-m k-1) !=\frac{n !}{m k(m k+1)} .
$$

Indeed, such a permutation $\pi$ satisfies

$$
\pi(j)<\pi(i+1)<\pi(m k+i+1) \quad i+2 \leq \forall j \leq m k+i .
$$


There are $\left(\begin{array}{c}n \\ m k+1\end{array}\right)$ ways to choose $P:=\pi([i+1: m k+i+1])$. Since $\pi([i+2: m k+i])$ can freely share the first $m k-1$ places of $P$, and since the complement of $P$ can be freely shared by other $n-m k-1$ numbers, we get (29).

Since case (d) occurs only when $m=p$, we similarly see that the number of $\pi$ 's satisfying (26) and (d) is given by

$$
\left(\begin{array}{c}
n \\
p k
\end{array}\right)(p k-1) !(n-p k) !=\frac{n !}{p k} .
$$

In the cases of $0 \leq i<r$, since $m k+i<n$ for any $1 \leq m \leq p, \delta_{i}^{+}$equals the total sum of $(29) / n !(m=1, \ldots, p)$. In the cases of $i=r$, since $m k+i$ equals $n$ only when $m=p, \delta_{i}^{+}$equals the total sum of $(29) / n !(m=1, \ldots, p-1)$ and $(31) / n !$. In the cases of $i>r$, since $m k+i<n$ for any $1 \leq m \leq p-1, \delta_{i}^{+}$equals the total sum of $(29) / n$ ! $(m=1, \ldots, p-1)$.

\section{A recurrence relation of $\left\{\phi_{i}\right\}$ : Case 3}

In this section, we consider the case of $k \leq i \leq\left[\frac{n-1}{2}\right]$. Then $i$ is expressed as

$$
i=q k+s
$$

for some $q \geq 1$ and $0 \leq s \leq k-1$. Since $i+k \leq n$, we get from Lemma 2.2 that $\left\{\operatorname{Ker}\left(i ; S_{\pi, i}\right) \mid i \in W_{S_{\pi, i}}, \pi \in \Pi\right\}$ is given by

$$
\left\{\begin{array}{rrrr}
{[i-k+1: i],} & {[i-k+1: i+1],} & \ldots & {[i-k+1: i+k-1]} \\
{[i-k+2: i+1],} & \ldots & {[i-k+2: i+k-1]} \\
& \ddots & \\
& & {[i: i+k-1]}
\end{array}\right\}
$$

and $\left\{\operatorname{Ker}\left(i+1 ; S_{\pi, i+1}\right) \mid i+1 \in W_{S_{\pi, i+1}}, \pi \in \Pi\right\}$ is given by

$$
\left\{\begin{array}{rrrr}
{[i-k+2: i+1],} & \ldots & {[i-k+2: i+k-1],} & {[i-k+2: i+k]} \\
& \ddots & \vdots & \\
& & {[i: i+k-1],} & {[i: i+k]} \\
& & & {[i+1: i+k]}
\end{array}\right\} .
$$

So, as well as (24), we have

$$
\begin{aligned}
\left\{\operatorname{Ker}\left(i+1 ; S_{\pi, i+1}\right) \mid i \notin W_{S_{\pi, i+1}}, i+1 \in W_{S_{\pi, i+1}}, \pi \in \Pi\right\} & \\
& =\left\{\left[j_{1}: i+k\right] \mid i-k+2 \leq j_{1} \leq i+1\right\}
\end{aligned}
$$

and

$$
\begin{aligned}
\left\{\operatorname{Ker}\left(i ; S_{\pi, i}\right) \mid i \in W_{S_{\pi, i}}, i+1 \notin W_{S_{\pi, i}},\right. & \pi \in \Pi\} \\
& =\left\{\left[i-k+1: j_{2}\right] \mid i \leq j_{2} \leq i+k-1\right\} .
\end{aligned}
$$


As well as (25), we get from (35) and (36) that

$$
\begin{aligned}
& \left\{I\left(i+1 ; S_{\pi, i+1}\right) \mid i \notin W_{S_{\pi, i+1}}, i+1 \in W_{S_{\pi, i+1}}, \pi \in \Pi\right\} \\
& \quad=\left\{\left[j_{1}: m k+i\right] \mid 1 \leq j_{1} \leq i+1, m \geq 1, m k+i \leq n\right\}
\end{aligned}
$$

and

$$
\begin{aligned}
\left\{I\left(i ; S_{\pi, i}\right) \mid i \in W_{S_{\pi, i}}, i+1 \notin W_{S_{\pi, i}}, \pi \in \Pi\right\} & =\left\{\left[i-m k+1: j_{2}\right] \mid i \leq j_{2} \leq n, m \geq 1,1 \leq i-m k+1\right\} .
\end{aligned}
$$

Lemma 6.1. Let $m \geq 1$ satisfy $m k+i \leq n$. Then there exists $i \leq j_{2} \leq n$ such that $I\left(i ; S_{\pi, i}\right)=\left[i-m k+1: j_{2}\right]$ if and only if

$$
\pi(j)<\pi(i) \quad i-m k+1 \leq \forall j \leq i-1
$$

and either (e) $\pi(i-m k)>\pi(i)$ or (f) $i-m k+1=1$ holds.

Proof. Almost same with Lemma 5.1. The only difference is that we make $I\left(i ; S_{\pi, i}\right)$ by attaching intervals of length $k$ to $\operatorname{Ker}\left(i ; S_{\pi, i}\right)$ from not the right side of $i$ but the left side of $i$.

TheOrem 6.2. In the case of $k \leq i \leq\left[\frac{n-1}{2}\right]$, it holds that

$$
\delta_{i}^{+}= \begin{cases}\sum_{m=1}^{p-q} \frac{1}{m k(m k+1)} & 0 \leq s \leq r-1, \\ \sum_{\substack{m=1 \\ p-q-1}}^{p-q-1} \frac{1}{m k(m k+1)}+\frac{1}{(p-q) k} & s=r, \\ \sum_{m=1}^{m k(m k+1)} \frac{1}{m+1 \leq s \leq k-1,} & r+1 \leq\end{cases}
$$

and

$$
\delta_{i}^{-}= \begin{cases}\sum_{m=1}^{q-1} \frac{1}{m k(m k+1)}+\frac{1}{q k} & s=0, \\ \sum_{m=1}^{q} \frac{1}{m k(m k+1)} & s \neq 0,\end{cases}
$$

where $q$ and $j$ are defined by (32).

Proof. One can easily prove (40) as well as (27). The only difference is that $p$ is replaced by $p-q$. The difference comes from that $i$ is expressed as $i=q k+s$. So, the condition $m \geq 1$ and $m k+i \leq n$ in (37) is equivalent to $m \geq 1$ and $(m+q) k+s \leq n$. When $s>r$, the latter implies that $1 \leq m \leq p-q-1$. When $s \leq r$, it implies that $1 \leq m \leq p-q$. In particular, when $s=r, m=p-q$ corresponds to (d) in Lemma 5.1.

We use (38) and Lemma 6.1 to prove (41). By Lemma 6.1, $\pi$ satisfies $i \in W_{S_{\pi, i}}$ and $i+1 \notin W_{S_{\pi, i}}$ if and only if $\pi$ satisfies (39) and either (e) or (f). The condition $m \geq 1$ and $1 \leq i-m k+1$ in (38) is equivalent to $1 \leq m \leq q$. In particular, when $s=0, m=q$ corresponds to (f). So we get (41).

Following is the graphs of the Shapley values of 28 players. 

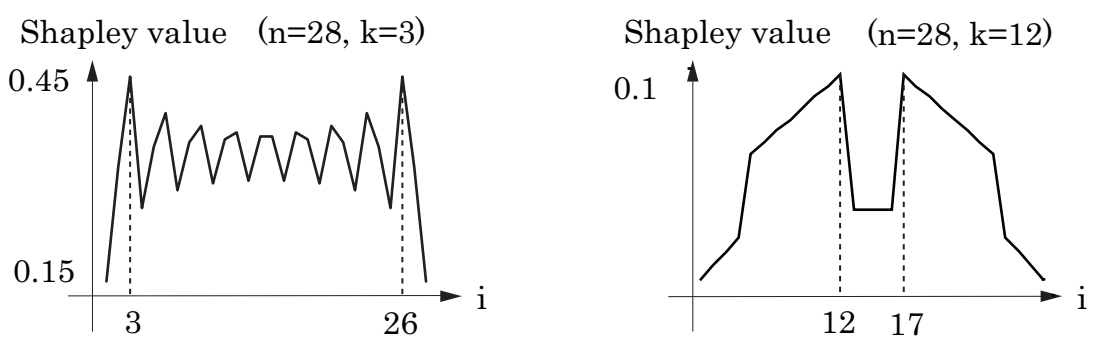

Figure 4: Shapley values of 28 players. Left: $k=3$, Right: $k=12$

\section{The maximal values of the Shapley value}

In Figure 4, the maximum value of the Shapley value is attained at $i=k$ and $i=n+1-k$. The aim of this section is to show that this is true for any $2 \leq k \leq n / 2$. Otherwise, the graph of the Shapley value has a shape in Figure 5.

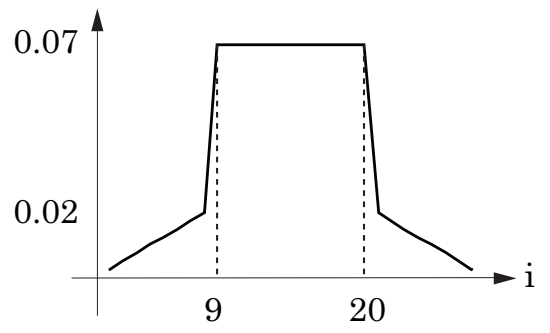

Figure 5: Shapley values of 28 players when $k=20$.

We first cosider the case of $k \geq n / 2+1$.

Theorem 7.1. When $k \geq n / 2+1$, it holds that

$$
\phi_{1}<\phi_{2}<\cdots<\phi_{n-k+1}=\cdots=\phi_{k}>\phi_{k+1}>\cdots>\phi_{n} .
$$

Proof. The assertion is a direct consequence of Theorems 4.2 and 5.2.

Next, we consider the case of $2 \leq k \leq n / 2$. We list up the maximal values of the Shapley value. By virtue of symmetry of the Shapley value, it suffices to consider $i \leq[(n-1) / 2]$, so that $i+1 \leq[(n+1) / 2]$. Since $n$ and $i$ are expressed as $n=p k+r$ and $i=q k+s, i \leq[(n-1) / 2]$ implies that

$$
(p-2 q) k+r-2 s-1 \geq 0 .
$$

Since $r \leq k$, we see from (42) that $p \geq 2 q$.

TheOREM 7.2. The maximal points of $\left\{\phi_{i}\right\}_{i}$ on the interval $[1:[(n+1) / 2]]$ are $\{k, 2 k, \ldots\}$. 


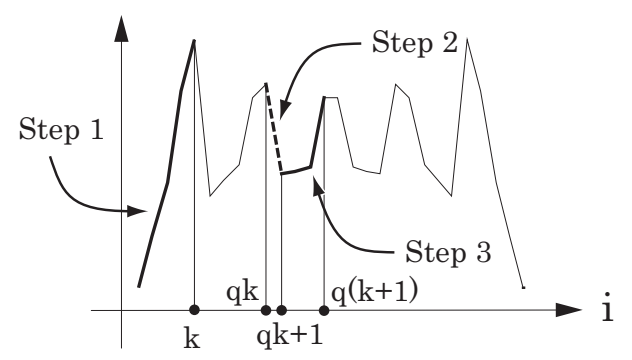

Figure 6: $\phi_{q k}>\phi_{q k+1} \leq \phi_{q k+2} \leq \cdots \leq \phi_{q(k+1)}$.

Proof. Figure 6 shows the outline of the proof. Step 1. It follows from Theorem 5.2 that $\phi_{1}<\phi_{2}<\cdots<\phi_{k}$. Step 2. We show

$$
\phi_{q k+1}-\phi_{q k}<0 \quad(q=1,2, \ldots) .
$$

We get from Theorem 6.2 that, for any $q=1,2, \ldots$,

$$
\phi_{q k+1}-\phi_{q k}= \begin{cases}\sum_{m=q}^{p-q} \frac{1}{m k(m k+1)}-\frac{1}{q k} & r>0 \\ \sum_{m=q}^{p-q-1} \frac{1}{m k(m k+1)}+\frac{1}{(p-q) k}-\frac{1}{q k} & r=0 .\end{cases}
$$

Indeed, since $s=0$ for $i=q k$, the first two cases in (40) and the first case in (41) are applicable, and we easily get (44). Further, we get (43) from (44). Indeed, for any $r>0$ and $p=2 q$, we have

$$
\phi_{q k+1}-\phi_{q k}=\frac{1}{q k}\left(\frac{1}{q k+1}-1\right)<0 .
$$

For any $r>0$ and $p>2 q$, we have

$$
\begin{aligned}
\phi_{q k+1}-\phi_{q k} & =\sum_{m=q}^{p-q-1} \frac{1}{m k(m k+1)}+\frac{1}{(p-q) k\{(p-q) k+1\}}-\frac{1}{q k} \\
& <\sum_{m=q}^{p-q-1} \frac{1}{m k m k}+\frac{1}{(p-q) k}-\frac{1}{q k} \\
& =\frac{1}{k}\left(\frac{1}{k} \sum_{m=q}^{p-q-1} \frac{1}{m^{2}}+\frac{1}{p-q}-\frac{1}{q}\right) .
\end{aligned}
$$

For $r=0$, we have from (44) that

$$
\phi_{q k+1}-\phi_{q k}<\frac{1}{k}\left(\frac{1}{k} \sum_{m=q}^{p-q-1} \frac{1}{m^{2}}+\frac{1}{p-q}-\frac{1}{q}\right) .
$$


So, letting $f(p):=\frac{1}{k} \sum_{m=q}^{p-q-1} \frac{1}{m^{2}}+\frac{1}{p-q}-\frac{1}{q}$, we see that

$$
0 \geq f(2 q+1)>f(2 q+2)>\cdots>f(p) .
$$

In fact,

$$
f(2 q+1)=\frac{1}{k q^{2}}+\frac{1}{q+1}-\frac{1}{q}=\frac{1-q(k-1)}{k q^{2}(q+1)} \leq 0
$$

and

$$
f(2 q+j+1)-f(2 q+j)=\frac{1}{k(q+j)^{2}}+\frac{1}{q+j+1}-\frac{1}{q+j}=\frac{1-(q+j)(k-1)}{k(q+j)^{2}(q+j+1)}<0 .
$$

Hence $f$ is nonincreasing, so that (43) has been proved. Step 3. We show $\phi_{q k+s} \leq$ $\phi_{q k+s+1}$ for any $s \geq 1$ and $q \neq 0$. (i) When $1 \leq s \leq r-1$, we see from the first case of (40) and the second case of (41) that

$$
\phi_{q k+s+1}-\phi_{q k+s}=\sum_{m=q+1}^{p-q} \frac{1}{m k(m k+1)} \geq 0,
$$

where the summation equals 0 when $p-q<q+1$. (ii) When $s=r$, it follows from the second case of (40) and the second case of (41) that

$$
\phi_{q k+s+1}-\phi_{q k+s}=\sum_{m=q+1}^{p-q-1} \frac{1}{m k(m k+1)}+\frac{1}{(p-q) k} \geq 0 .
$$

(iii) When $s>r$, it follows from the third case of (40) and the second case of (41) that

$$
\phi_{q k+s+1}-\phi_{q k+s}=\sum_{m=q+1}^{p-q-1} \frac{1}{m k(m k+1)} \geq 0 .
$$

Therefore $\phi_{q k+s+1} \geq \phi_{q k+s}$.

Theorem 7.3. When $2 \leq k \leq n / 2$, the maximum points of $\left\{\phi_{i}\right\}_{i}$ are $i=k$ and $i=n-k+1$.

Proof. By Theorem 7.2, the maximum value is attained by either $i=q k$. In the case of $r=0$, it follows from the second case of (44) and (49) that

$$
\phi_{(q+1) k}-\phi_{q k}=\sum_{s=0}^{k-1}\left(\phi_{q k+s+1}-\phi_{q k+s}\right)=k \sum_{m=q+1}^{p-q-1} \frac{1}{m k(m k+1)}+\frac{1}{(p-q) k}-\frac{1}{q k+1} \text {. }
$$

Here, remark that the summations in (44) and (47) are taken from $m=q+1$ to not $p-q-1$ but $p-q$. So, in the case of $r>0$, it follows from the first case of (44), (47), (48), and (49) that

$$
\begin{aligned}
& \phi_{(q+1) k}-\phi_{q k}=\sum_{s=0}^{k-1}\left(\phi_{q k+s+1}-\phi_{q k+s}\right) \\
= & k \sum_{m=q+1}^{p-q-1} \frac{1}{m k(m k+1)}+\frac{r}{(p-q) k\{(p-q) k+1\}}+\frac{1}{(p-q) k}-\frac{1}{q k+1} .
\end{aligned}
$$


Since (51) reduces to (50) when $r=0,(51)$ is correct for $r=0$.

For $1 \leq q, 2 q+2 \leq p, 0 \leq r \leq k-1$, and $2 \leq k$, let $f_{1}(p, q, r, k):=\phi_{(q+1) k}-\phi_{q k}$. Then, it is obvious that $f_{1}(p, q, r, k) \leq f_{1}(p, q, k-1, k)=: f_{2}(p, q, k)$ for any $0 \leq r \leq k-1$. Further,

$$
f_{2}(p+1, q, k)-f_{2}(p, q, k)=\frac{-(k-1)}{(p-q+1)\{(p-q+1) k+1\}\{(p-q) k+1\}}<0 .
$$

Hence $f_{2}(p, q, k)$ is the strict decreasing w.r.t. $p$. So, let $f_{3}(q, k):=f_{2}(2 q+2, q, k)$. Then

$$
\begin{aligned}
f_{3}(q, k) & =\frac{1}{(q+1)\{(q+1) k+1\}}+\frac{k-1}{(q+2) k\{(q+2) k+1\}}+\frac{1}{(q+2) k}-\frac{1}{q k+1} \\
& =\frac{-2 q^{2} k^{2}+2 q^{2} k-5 q k^{2}+3 q k-4 k^{2}+2 q+k+3}{(q+1)(q+2)(q k+1)(q k+k+1)(q k+2 k+1)} .
\end{aligned}
$$

Since the numerator of the right-hand side is expressed as

$$
-(k-1)\left\{2 k\left(q+\frac{5 k+2}{4 k}\right)^{2}-\frac{(5 k+2)^{2}}{8 k}+4 k+3\right\},
$$

the maximum value of $f_{3}(q, k)$ on $q \geq 1$ is attained by $q=1$. Then the numerator of $f_{3}(1, k)$ is $-(k-1)(11 k+5)<0$. So, $f_{1}(p, q, k, r) \leq f_{2}(p, q, k) \leq f_{3}(q, k)<0$ as desired.

\section{Acknowledgement}

The authors would like to thank the referee for valuable comments. This research was supported by JSPS KAKENHI Grant No. 23540142.

\section{References}

Aumann, R. J., and Hart, S. (1992). Handbook of game theory with Economic Applications Vol. 1, Elsevier, Amsterdam.

Gelfand, I. M., and Fomin, S. V. (1963). Calculus of variations, Prentice Hall.

Kawasaki, H. (2000). Conjugate points for a nonlinear programming problem with constraints, Journal of Nonlinear and Convex Analysis 1, 287-293.

Kawasaki, H. (2001). A conjugate points theory for a nonlinear programming problem, SIAM Journal Control and Optimization 40, 54-63.

Kawasaki, H. (2003). Analysis of conjugate points for constant tridiagonal Hesse matrices of a class of extremal problems, Optimization Methods and Software 18, 197-205.

Kawasaki H. (2004). A game-theoretic aspect of conjugate sets for a nonlinear programming problem, Proceedings of the third International Conference on Nonlinear Analysis and Convex Analysis, Yokohama Publishers, 159-168.

Kawasaki H. (2005). , Conjugate-set game for a nonlinear programming problem, in Game theory and applications 10, eds. L.A. Petrosjan and V.V. Mazalov, Nova Science Publishers, New York, USA, 87-95. 
Shapley L. S. (1953). A value for N-person games, in Kuhn and Tucker (eds.) Contributions to the Theory of Games, Vol. II, 305-317.

Received June 17, 2015

Revised August 21, 2015 